\title{
ON AN OUTBREAK OF SCARLET FEVER AND SCARLATINAL SORE THROAT DUE TO INFECTED MILK*.
}

BY ARTHUR NEWSHOLME, M.D.

Medical Officer of Health of the Borough of Brighton.

$\mathrm{N}$ October last an interim report was made concerning an outbreak
of scarlet fever and scarlatinal sore throat, associated with a particular milk supply. The administrative measures which were taken were fortunately successful in preventing extension of the epidemic, and as they were largely independent of statutory powers, it appears desirable to record the circumstances for future guidance.

For the right understanding of the epidemic, it is convenient to summarize certain facts as to the spread of infective diseases. Such diseases are, probably in every instance, due to a specific microbe, the illness being caused by the poisonous products of the growth of the microbe. These products may be manufactured by the microbe in the blood or tissues of the patient, or they may have been prepared in advance by the growth of the microbe in some other substance. In either case, the probability of producing disease and the severity of the attack depends partly on the virulence or poisonous qualities of the microbe to man, partly on the dose of microbes received, and partly on the susceptibility of the patient. In scarlet fever, as well as in other microbic diseases, a difference arises according as the microbe elaborates its poison in the body of the patient, or is prepared in advance in some other medium. In the former case the incubation period is usually more prolonged before the poison has accumulated sufficiently to overcome the natural resistance of the patient and recognizable symptoms of scarlet fever appear. In the latter case, when the microbe has lived in a suitable substance, it may elaborate in that substance doses of poison large enough to cause recognizable symptoms within a few hours after this substance has been taken into the patient's body. Clinical evidence shows that the microbe of scarlet fever grows readily in milk; and the subsequent distribution of scarlet fever produced by this milk would obviously be much more rapid and extensive than could possibly occur in connection with personal infection, being limited in fact only by the distribution of the milk. Personal infection passes slowly from person to person, infection by milk acts on the same day on a large proportion of the total number consuming the milk in question. There are thus, at

\footnotetext{
* From Annual Report, Brighton, 1906.
} 
least, two differences between epidemics of scarlet fever due to milk infection and those due to personal infection. (1) In milk epidemics the incubation period in each attack is-most often extremely short; and (2) the total epidemic is explosive in character ; while in epidemics due to personal infection, the incubation period of each individual attack is somewhat longer, and the total epidemic is much more prolonged. In one instance the epidemic is usually a question of a few days, in the other of weeks, or more often of months.

These facts are stated, not for their theoretical interest, but because they give certain guiding rules for preventing the spread of scarlet fever, of which the most important are that when cases of scarlet fever and scarlatinal sore throat appear in suddenly increased and rapidly increasing numbers, first the milk supply should be promptly and carefully investigated; and secondly, inasmuch as the delay of a few hours is competent to increase an epidemic to a disastrous extent, action must at once be taken on the best evidence that is available, irrespective of whether that evidence is conclusive as to the cause of the outbreak, so long as it justifies a reasonable apprehension of public danger.

It follows from these rules that in dealing with an outbreak of scarlet fever any procedure involving delay through legal or administrative routine is dangerous to the public safety, and that when this disease is conveyed by milk the community is dependent largely for its control on the fulness and promptitude of the information obtainable from the dairyman. In view of this circumstance, in November, 1905, I issued to the dairymen of Brighton a circular, of which a copy is appended to this report, inviting them to keep a constant record of the separate sources of the milk distributed daily on each round; and this recommendation has been largely adopted, and has been so adopted in the dairy in which the present outbreak occurred, thus enabling me immediately to identify the source of the milk supplied to the implicated households.

The first two cases of scarlet fever in the present epidemic were notified on October 15th, and on the same evening the inspector's routine report stated, among other things, that the families were supplied with milk from A.'s dairy. Although these two cases were totally insufficient to justify a definite conclusion, my suspicion was aroused by the fact that several "swabs" from suspected diphtheria patients had arrived during the same day; scarlet fever in its early stages presenting symptoms often difficult or impossible to distinguish clinically from diphtheria. I at once telephoned to the dairy, and found that it supplied the milk to each of the houses from which the 
"swabs" had come, as well as the two houses where scarlet fever had occurred. These facts, although they made me apprehensive, did not justify immediate action in regard to the milk; but from this point every doctor seen was recommended to advise the temporary boiling of milk among his patients. Next morning (16th October) at 9.0 a.m., I was informed by a doctor that he had several cases of sore throat in his practice. Particulars of these were obtained; and when the manager of the dairy later in the day called on me by previous appointment, I found that in these cases not only had their milk come from the dairy A., but also from B.'s farm, whence the chief part of A.'s milk was received. The facts so far collected, conjoined with those relating to the boarding school $Z$., which had meanwhile come into my possession, justified the conclusion that the outbreak was attributable to this source, and the action which this conclusion demanded. A special meeting of the Sanitary Committee was called for the following morning (17th October). In the meantime, I went over the facts with three representatives of the dairy; and the farmer suspended from his work a milker to whose circumstances suspicion attached. The Committee authorized the payment to the farmer of partial compensation at the rate of $4 \mathrm{~d}$. per gallon, in consideration of his destroying all his milk for a week, and this arrangement was extended subsequently for a second week. With this measure the epidemic stopped. The total amount paid to the farmer was $£ 9310 \mathrm{~s}$.

These facts in themselves show that the epidemic was eaused by the milk in question, and it is indeed seldom that a sanitary authority has, in such cases, so high a degree of probability on which to base its action. In controlling the epidemic within two days of the notification of the first cases the measures taken at each stage were only possible through the co-operation of the dairyman. $\mathrm{Had}$ it been necessary to proceed by compulsory powers, some days, and perhaps a week, would have elapsed in the routine procedure involved; and, if the infection had persisted, the dimensions of the epidemic would have been enormously larger than the considerable numbers which actually occurred. It is extremely difficult to prevent accidental infection even in a dairy, in which, as in Dairy A., the management is good; and it is thus most important for the town to show to the dairyman the utmost consideration consistent with its safety; and even after the event to show on the basis of the facts of the entire epidemic as it eventually became known that the original induction was completely accurate.

Of course this does not imply that even if fuller knowledge had 
shown that all the previous coincidences between the supply of milk from B.'s farm and the cases of scarlet fever, in association with the absence of scarlet fever where this milk was not supplied, and with the striking experience of the boarding school $Z$., were accidental and not significant, the action of the Sanitary Committee would thereby have been shown to be improper. In the case of a rapidly spreading disease it is as important to the community that what is reasonably apprehended to be a source of infection should be stopped, as it is to a family that a suspected case of infection should be isolated; and

Table I.

NUMBER OF CASES OF SCARLET FEVER AND SORE THROAT AMONG CUSTOMERS OF A.'S DAIRY IN THE BOROUGH AND IN THE REST OF THE BOROUGH.

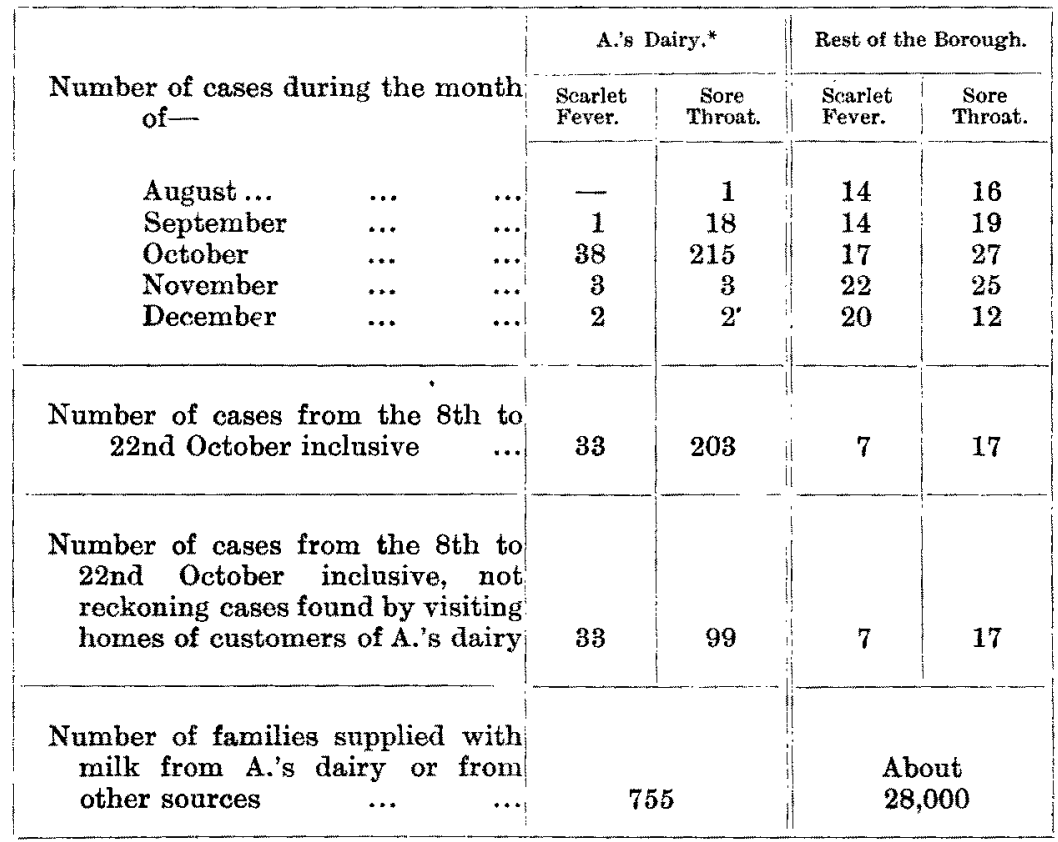

the action taken must be that which will minimize danger, if the reasonable apprehension is subsequently confirmed. In most epidemics the early evidence is not so clear as in this case, and the fuller information necessary to incriminate a milk supply with certainty must consist of the actual continuance of the epidemic ; and it is therefore in such instances inconsistent with public safety to delay action in the face of reasonable apprehension, even though conclusive evidence

\footnotetext{
* Including 5 cases of scarlet fever among customers of U.'s dairy.
} 
may not be available. If, however, the co-operation of dairymen, on which the rapid suppression of these dangerous and costly epidemics depends, is to be secured, they can reasonably expect that the fuller facts of the entire epidemic shall be investigated and set forth, in which the evidence as to cases known when administrative action was taken and those subsequently coming to light shall be sifted and stated. In the following pages this has been done.

The accompanying coloured chart (Fig. 1) shows for the month of October, in which the epidemic occurred, and for the two previous and two subsequent months, the number of cases of scarlet fever and of sore throat which occurred $(a)$ among the families supplied with milk from the incriminated dairy, and (b) in the rest of the borough. In a lower part of the same chart are shown the cases of scarlet fever and of sore throat occurring outside the borough among customers of the dairy in question which came to my knowledge.

For obvious reasons I cannot supply corresponding data for the remaining parts of the population of these districts outside the borough. Each primary case of scarlet fever is shown in Fig. I. by a red square, and of sore throat by a black square; secondary cases, infected from previous patients in the same outbreak, being shown by circles. The same facts, classified according to months, are set forth in Table I., so far as relates to the borough. In regard to all these figures, which represent the dates of probable onset of the disease, it must be borne in mind that they cannot be stated within a day or two with actual certainty. In the charts and tables they have been taken at the earliest probable date.

Sources of Information.-Information as to cases was derived from the following sources:-

1. Notifications of cases of scarlet fever.

2. Informal notification by doctors of cases of sore throat, which were not recognizable as scarlet fever. Many doctors consulted me as to puzzling cases under this heading which had occurred among their patients; and by this means, as well as from official sources, I received information as to illness outside the borough. Notification as to similar cases of sore throat reached me through the following further channels.

3. A certain number of cases were notified as diphtheria, but were found not to have diphtheria-bacilli in their throats, and were associated with the outbreak of scarlet fever.

4. In other cases diphtheria was suspected, and throat-swabs were sent to me for examination, with a negative result. The clinical diagnosis of diphtheritic from scarlatinal throats is often very difficult. 


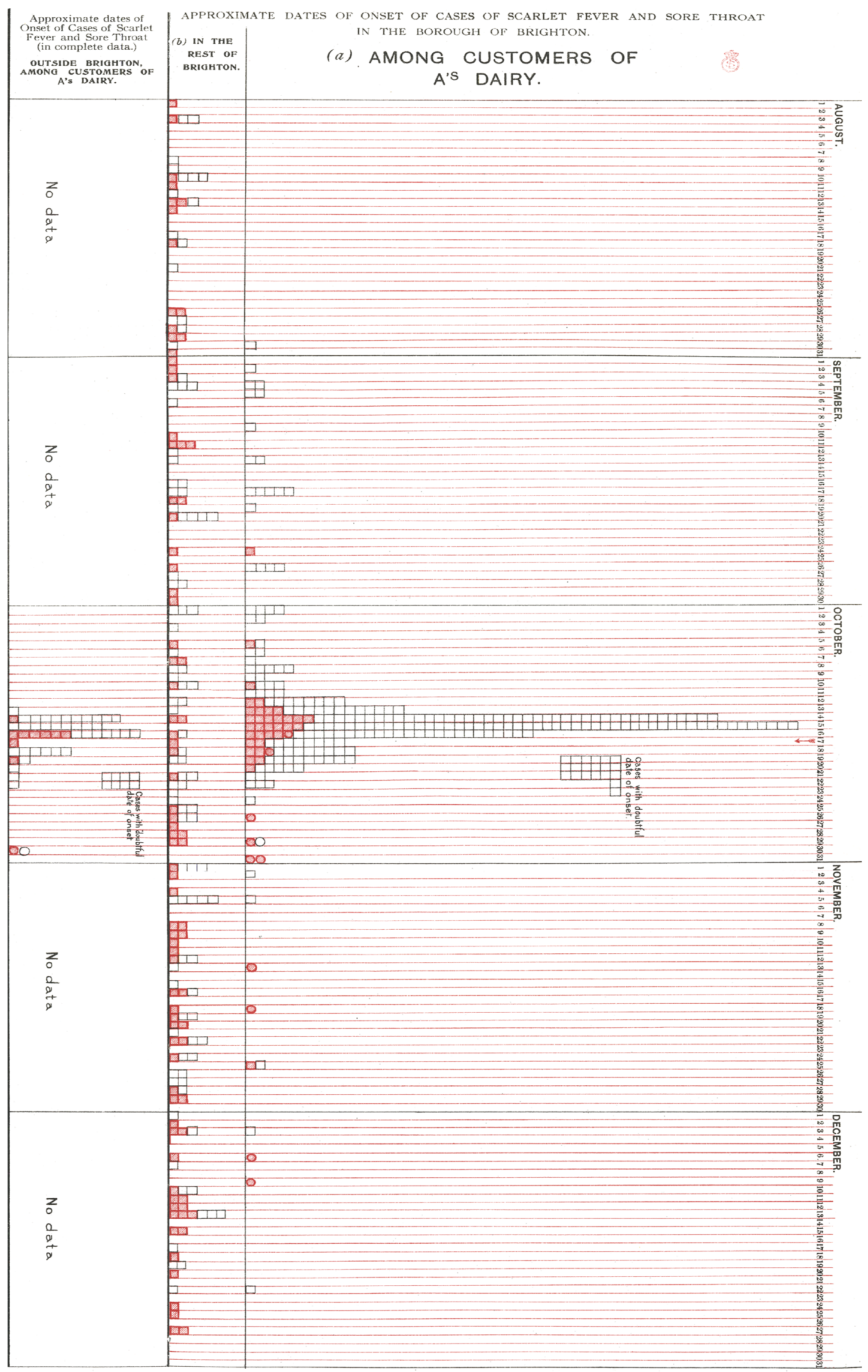


5. When the outbreak was fully established, and very numerous messages and letters were being received from both inhabitants and doctors on the subject, the opportunity was taken to make a house-tohouse enquiry among the customers of A.'s dairy.

At this enquiry the date of onset of any cases of sore throat was ascertained, and no mention of milk supply was made. These enquiries were made after the supply from A.'s farms had been stopped; and the opportunity was taken, when suspicion had been already aroused, to state that the then supply of milk was quite safe. One hundred and four cases were ascertained in this way, and precautionary measures were thus rendered practicable which, had they not been taken, might have been followed by a considerable crop of secondary cases. There can be little doubt that the information obtained by these different means understates the full magnitude of the epidemic.

Looking back at the facts gradually collected by these various means, it is evident that some cause of scarlet fever and sore throats came into rather sudden and extensive operation during the month of October.

\section{TABLE II.}

NUMBER OF CASES OF SCARLET FEVER AND SORE THROAT IN THE BOROUGH PER 100 FAMILIES HAVING OR NOT HAVING MILK FROM A.'S DAIRY.

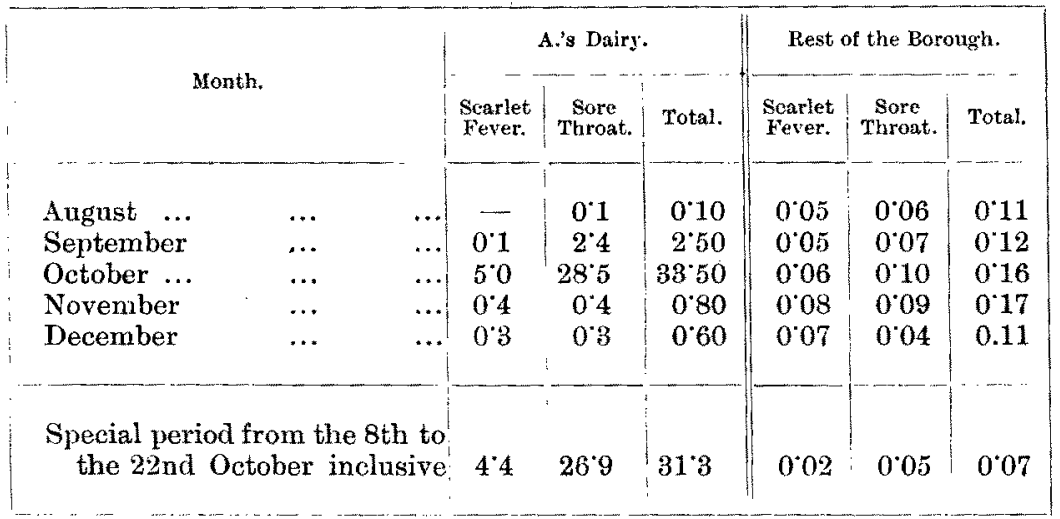

Evidence of Milk Infection.-1. That this was no ordinary spread by personal infection is almost proved by inspection of the chart. It is, as is pointed out above, characteristic of scarlet fever that when spread by personal communication it spreads slowly and gradually. The present outbreak was explosive in character. Such peaks as that in the chart are never seen in the experience of a large town 
762 Scarlet Fever \& Scarlatinal Sore Throat [Publc Health

Fraure II.

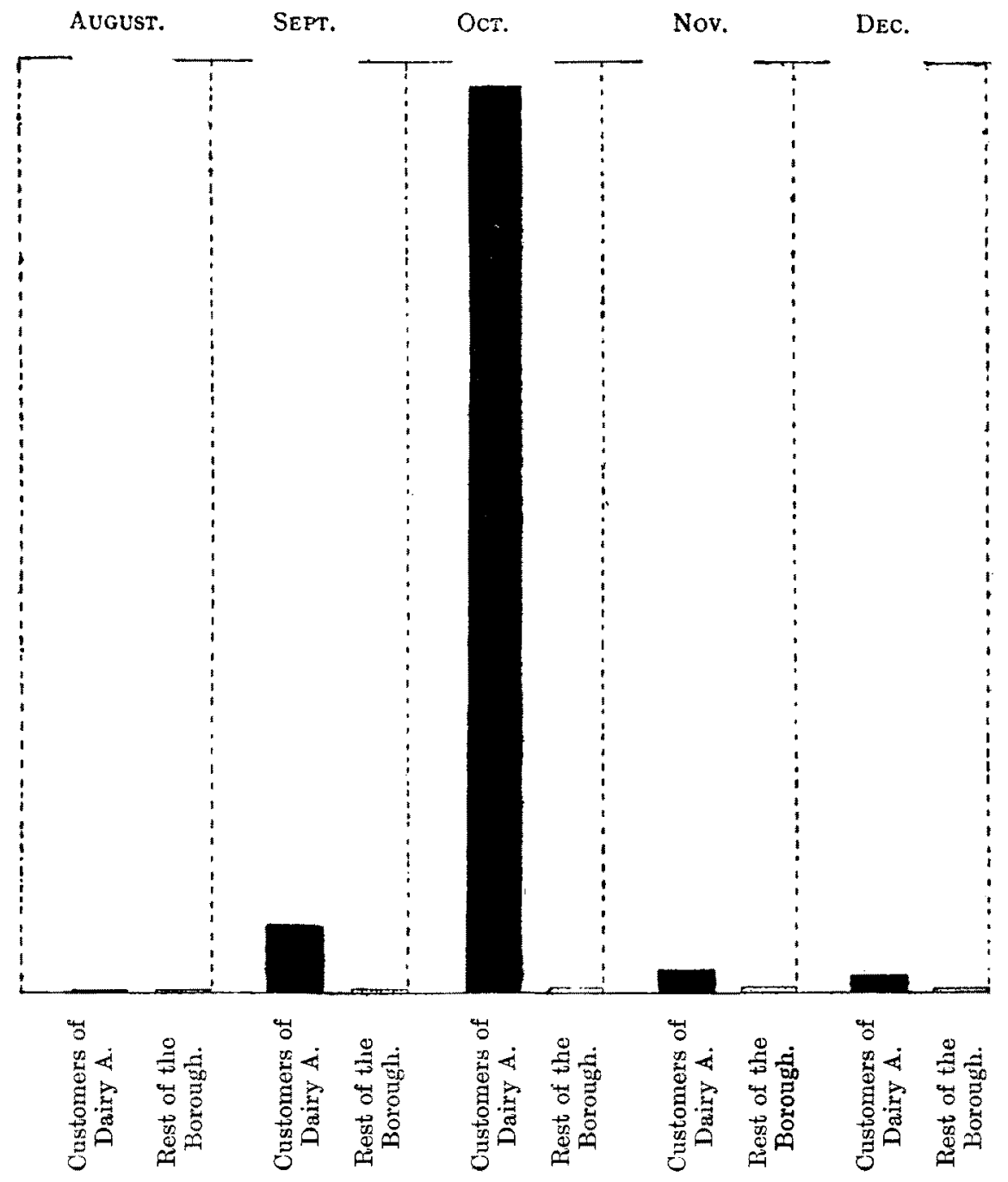

Diagram showing the relative incidence of Scarlet Fever and Scarlatinal Sore Throat in every 100 houses.
(a) Among customers of A.'s Dairy.
(b) In the rest of the Borough. 
unless a water supply has become infected on a large scale as in enteric fever, or a milk supply as in scarlet fever.

2. That the milk of A.'s dairy caused the chief outbreak is proved by the chart relating to the borough, in inspecting which it must be remembered that the upper part relates to 755 , and the lower to 28,000 households. This is shown more clearly when the incidence of sickness is stated in proportion to every 100 families, as in Table II. The differences there indicated are set forth graphically in Fig. II. The immense preponderance during October of sore throats and scarlet fever in connection with the milk supply is very obvious.

3. The social and domestic circumstances of the vast majority of the patients excluded personal infection as a primary cause of the epidemic. Of the total 236 cases of scarlet fever and sore throat occurring in the borough between the 7 th and $23 \mathrm{rd}$ of October among customers of A.'s dairy, six of scarlet fever and twenty-three of sore throat occurred in six boarding schools and small homes connected with schools. The occupants of these establishments live a relatively segregated life, and their movements can be traced with exactitude. Exhaustive enquiries were made, and in not a single instance could the importation of infection be traced, apart from milk. A similar investigation showed that in the private families involved similar personal infection was very improbable. The chart shows that at the time when the epidemic occurred among the customers of A.'s dairy, there was for a population of about 125,000, only a small amount of scarlet fever in the rest of the town.

The special circumstances of several cases almost certainly excluded personal infection. Thus, attacks occurred in bedridden patients, and in children who had been confined to the house for a fortnight.

4. The geographical distribution of the cases followed the distribution of the milk from A.'s dairy over two towns and to a boarding school which was outside both towns. Complete information as to locality of cases can only be given for the borough itself. Various parts of the borough were dotted with cases of illness in fair accordance with the amount of milk distributed by $A$. in these parts. The incomplete information available for the areas of supply outside the borough (shown graphically in the lower part of the chart) indicates that the distribution of illness in these parts, constituting about a third of the total supply, was similar to that in the borough. In arriving at this conclusion, it has to be borne in mind that 104 cases were discovered in the borough by means of domestic enquiries, which could not be extended beyond the limits of the borough. 


\section{Scarlet Fever \& Scarlatínal Sore Throat [Public Healtn}

5. Control circumstances were furnished by some families. The following five instances may be quoted:-

(1). A family supplied with milk from A.'s dairy consisted of five adults and one child. Their milk was always boiled, except for the nurse, who would not drink milk except unboiled. The nurse had a severe sore throat, which was diagnosed as diphtheria, but proved not to be ; all the rest of the family escaped.

(2). A certain family receiving milk from A.'s dairy made a practice of boiling it. For two days at the time of the epidemic, owing to domestic difficulties, this was intermitted, and on the third day a delicate boy in the family fell ill with severe sore throat.

(3). A children's hospital with fifty-eight beds is supplied with milk from A.'s dairy. All the milk supplied to the patients at this hospital is sterilized, and not a single case of scarlet fever or sore throat occurred among them at the time of the epidemic.

(4). A boarding school with ten boarders was supplied with A.'s milk, which was always boiled. No cases of sore throat without rash, and only one case of scarlet fever occurred at this school. This patient fell ill on the 16th October. On the 15th she was in contact with a day scholar, D. S., from a neighbouring district, who then had a sore throat, and who drank A.'s milk unboiled at home. It may be added that on the 19th November D. S. returned to school. A day scholar conversed with her that afternoon, and about thirty hours later developed scarlet fever.

(5). At the boarding school Z., six cases of searlet fever and four of sore throat occurred among forty-five resident pupils. These cases of illness occurred solely among boys on "special milk," which means that they had a pint of milk each day. Not a single case occurred among the boys who only had milk in tea, coffee, puddings, etc.

The cumulative force of the evidence that the milk from A.'s dairy caused the explosive outbreak of illness among the customers of the dairy is irresistible. This being so, it next becomes necessary to examine the milk supply.

Contamination of Milk in Transit.-The milk supplied from A.'s dairy might have become contaminated in transit or at its source. The wide distribution of the cases of sore throat and scarlet fever over different and remote districts of and outside the borough, showed that many milk rounds were implicated, and a detailed investigation of the family circumstances and health of the twenty men and boys employed by this dairy, and of the employees at the dairy itself, and the depôt connected with it, failed to discover a single case of illness preceding the outbreak. 
Dual supply of Mitk.-A.'s dairy was found to be supplied daily from two sources ; about 370 gallons a day from B.'s farm just outside the borough, and about 68 gallons from T., about 200 miles distant. Which of these was the source of infection, or were both? At the time when it became my duty to decide this question, I only knew of seventeen cases of illness among the customers of A.'s dairy, of which seven were scarlet fever and ten sore throat. The manager of A.'s dairy, as stated above, had complied with the general request made in my official circular to dairymen, and was able to tell me the source of the milk supplied to each of the above seventeen patients, and if required, to each of the 755 households supplied by the dairy in, and the 330 households outside, the borough. On the strength of the information respecting these seventeen cases, I decided that the infection was derived from the neighbouring farm of B., with a probability sufficient to demand action.

Evidence that the Mill from B.'s Farm was the source of Infection.Of the seventeen cases of illness then known, five received milk from $B$., eleven from $B$, and $T$. (one in the morning and the other in the afternoon round), and one from a dairyman U., who receives part of his milk from B. and part from other sources. Excluding for practical purposes the highly improbable supposition that three separate supplies of milk (B., T. and the independent portion of U.'s supply) were simultaneously supplying infected milk, it is evident that B. is the only factor common to all the cases, and that $\mathrm{B}$. was the fons et origo mali. On this evidence B.'s milk supply was promptly stopped and the epidemic stopped with equal promptitude.

At two later stages I was able to check and confirm this early decision, on the cases of scarlet fever and sore throat notified, though not originating, after the milk supply from $B$. had been stopped. The three consecutive results are set out in the following table :-

TABLE III. (See p. 766\%.)

DISTRIBUTION OF CASES OF SCARLET FEVER AND SORE THROAT ACCORDING TO SOURCE OF MILK SUPPLY.*

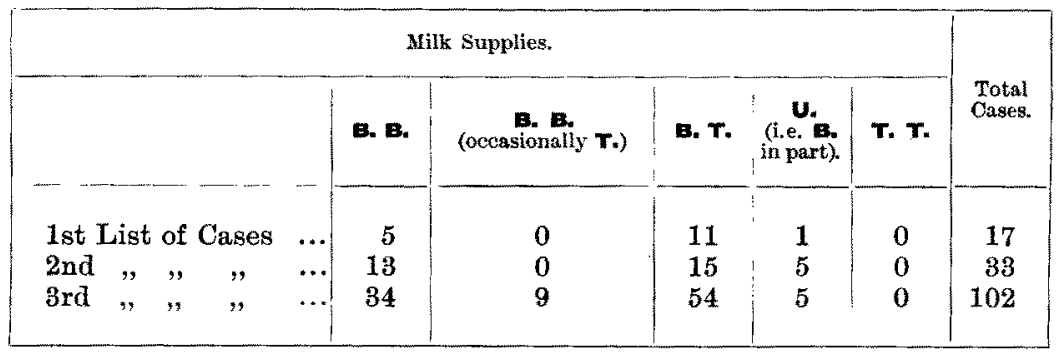


It will be noted that in each successive series of cases (1) B.'s milk was supplied wherever cases of sickness occurred; (2) milk from $T$. was absent in the first series from 35 per cent, and in the second and third series from at least 39 per cent of the families supplied with and suffering from the effects of contaminated milk. It follows certainly that the milk from T. had no causative relationship to the entire outbreak, and almost certainly played no part in causing the outbreak.

That the milk from B.'s farm caused the outbreak is shown by a number of further circumstances, one of which amounts to what in scientific investigations is known as a control experiment. This will be described later as a "control experience."

(1). Experience as to definite cases of scarlet fever.-During the month of October thirty-two cases of scarlet fever occurred in the borough among customers of A.'s dairy, and six further cases infected secondarily by personal infection. Six further cases occurred in the boarding school $\mathrm{Z}$. The facts as to source of milk supply can be given with exactitude for these thirty-eight primary cases of scarlet fever, and they are set forth in tabular form as follows :-

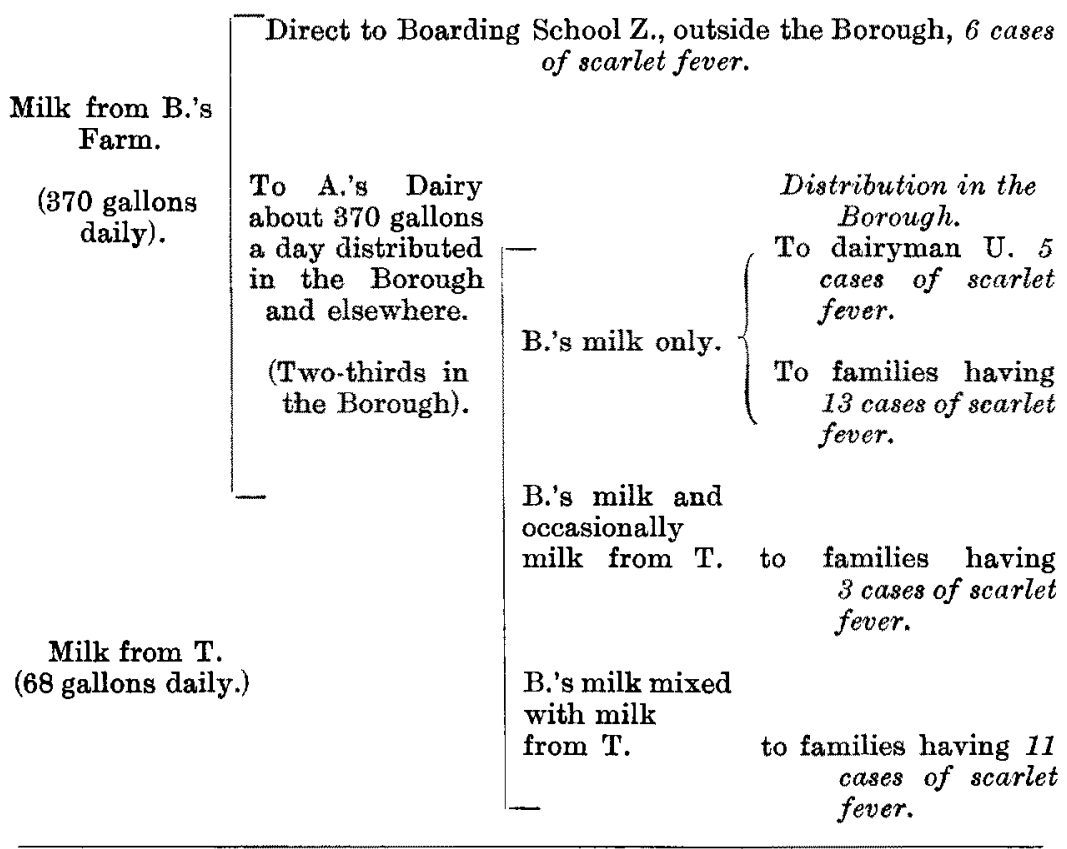

* The facts in Table III were compiled from information from the manager of A.'s dairy. At first this information was given without any reservation. Subsequently he stated that the above grouping of supplies could not be absolutely depended upon, and that in the rounds supplied with B.'s milk, a small amount of milk from $T$. may occasionally have been distributed. The substantial accuracy of the information originally supplied by him is, however, proved not only by the preceding facts, but also by those contained in the next paragraph. 
(2). Experience as to cases of sore throat.-The information at my disposal does not enable me to give corresponding information relating to the 215 cases of sore throat occurring in the borough during October among customers of A.'s dairy. It has been possible to obtain it for 102 cases of illness with the consistent result shown in Table III.

(3). The experience of the dairy $U$. confirms the same conclusion. This dairyman supplied 150 gallons of milk daily to his customers, and at the time of the epidemic was purchasing from A.'s dairy ten to twenty-five gallons of milk daily. This milk came from B.'s farm and was unmixed with milk from $T$. It was distributed in a special localized district, and no cases of scarlet fever occurred among U.'s customers except in this localized district, in which five cases occurred simultaneously with the cases among the direct customers of A.'s dairy. There were no other cases of scarlet fever in this localized district at the time.

(4). The experience of the boarding school $Z$. gives a conclusive "control experience." This school has forty-five resident pupils, among whom six cases of scarlet fever and four of sore throat occurred from the 13th to the 19th of October inclusive. The first patient was a boy with a sore throat, who was strictly isolated from the 13th to the 15th of October. On the latter day a second boy fell ill with sore throat, on the 16th four boys failed with scarlet fever and one with sore throat, on the 17 th one boy failed with scarlet fever, and on the 19th one case of scarlet fever and one of sore throat occurred. The first patient had not been exposed to any outside source of personal infection, and the isolation arrangements were so good as to render it improbable that this first patient could have infected the nine subsequent patients. The ten patients were distributed over three dormitories, and the patient who fell ill next after the first slept in a separate dormitory. After the first few days, these ten patients were all transferred to a special ward in our Borough Isolation Hospital, where they were all nursed together. To imagine that ten patients would thus rapidly be infected from one another in an admirably organized boarding school, under daily medical supervision and control, is contrary to everything known about the conditions of spread of scarlet fever. That such an explanation would be erroneous is further indicated by the contemporaneous events in the neighbouring borough, including six other boarding schools, which are shown in the chart. During the same days on which the boys at the school $\mathrm{Z}$. were falling ill, numerous similar cases were beginning in many different districts, all having one factor, and one factor only in common, that they were supplied with milk from A.'s dairy or B.'s farm. 
The relationship of the school $Z$. to this dairy was peculiar. The headmaster of the school, in his anxiety to secure the best possible milk supply for his pupils, had a rigid agreement that he should be supplied with milk in a sealed and locked four-gallon can, this milk being always put into this can at the farm B., and never mixed with any other milk at the depôt connected with A.'s dairy. There can be no doubt that this agreement was never in default, and this particular arrangement, therefore, forms conclusive evidence, if further evidence were required, that the infectivity of the milk of A.'s dairy originated on B.'s farm. The special incidence of illness among boys at this school having an extra milk diet has already been stated.

Circumstances of B.'s Farm.- - It has been conclusively shown that the milk supplied by A.'s dairy was not contaminated by its auxiliary supply from T., but on the way from B.'s farm to the dairy depot or actually on the farm. The contamination of the milk in transit was extremely improbable for all the churns, and impracticable so far as the supply to $Z$. was concerned. The carter was examined, with a negative result, and there was no illness in his family.

B.'s dairy farm is kept under conditions as to cleanliness and sanitary supervision which are much above the average. Each milker is under an obligation to report any suspicious case of illness. At the time of the outbreak 148 cows were kept. These were milked by eighteen men in the morning, and ten in the afternoon.

How did the Milk on B.'s Farm become infected?-Milk is known to produce scarlet fever when those suffering from this disease or possibly those coming from infected households are brought into close contact with it, as in milking. It is also believed that a certain disease of cows, the "Hendon disease," may sometimes cause scarlet fever in human beings consuming unboiled milk from the affected cows. These two lines of investigation were taken up. It should be stated at this point that the farmer gave every facility for inspection by myself or my deputy, of the cowsheds and of all employees and of the cows by the veterinary surgeon of the Corporation. This is specially mentioned, because, as I have already pointed out, had we been obliged to wait the adoption of the legal procedure under Section 4 of the Infectious Disease Prevention Act, considerable delay in the investigations at the farm would have arisen. In actual fact I was previously familiar with the cowsheds and cottages on this farm, which are much more sanitary and better kept than the majority of similar premises on farms.

(J). As regards employees and their families, these were all examined on several occasions by several doctors without finding definite evidence 
of illness. Two cowmen, father and son, were suspected to be desquamating, but I was unable on examining the men to confirm the suspicion, and apparently they had not been ill. The child of another cowman fell ill during the epidemic with supposed scarlet fever and was removed to an isolation hospital. Whether this child was infected from the milk, or her father had previously had an unrecognizable attack of scarlet fever could not be certainly stated. The child of a third cowman P., near the end of July, was removed from a cottage on the farm to an isolation hospital suffering from supposed scarlet fever. Two or three weeks later while in hospital she developed a very severe attack of scarlet fever. It is possible that the first attack was mistakenly thought to be scarlet fever, and that she caught this disease in hospital; it is equally possible that the second attack was a relapse. At the time of writing I have under my care a case of such relapse of scarlet fever, in which the relapse is much more severe and complicated than the undoubted first attack of scarlet fever for which the patient was admitted to hospital.

At the time when the milk at B.'s farm became infected, the preceding child was still in hospital. She had been visited weekly by her father, who had been back at his milking work for several weeks at the time of the milk-produced epidemic. There is every reason to think that he could not have brought home infection from the hospital, and apparently he himself had remained well. On September 2nd another child of this cowman became feverish, after visiting the hospital earlier in the same day. A doctor saw the child the same evening and again the next day. The possibility of scarlet fever was canvassed, and after some hesitation excluded. During the three weeks preceding the milk-produced epidemic, this child played in the cowsheds on different occasions. It should be added that on December 2nd, this child went to the village of $S$. to visit his sister, who by this time had left the hospital, and on the 4 th of December he fell ill with scarlet fever.

From the preceding sketch of facts, it is evident that one cannot with any certainty state a definite human medium by which the milk became infected. The evidence stops at the stage of suspicion and surmise, and does not throw any blame on anyone concerned; though I think we may draw the moral that cowmen's children should not be allowed to visit, and that their homes should not be near cowsheds.

(2). As regards cows, the veterinary inspector reported on the 18th October, that a cow had a pustule on its udder, but that this was not, in his opinion, of the same nature as those present in the "Hendon disease." Unfortunately in the rush of work, the opportunity 
of obtaining cultures from this pustule was lost. The cows were also seen by the superintendent of the Brighton Public Abattoir, and he reported that one cow which with seven others had come from M. C., on October 6th, had an abscess on the udder, with a scab over it. This is the same case as the one reported by the veterinary inspector, and subsequently got well. Another cow had a small abscess of the udder, and was slaughtered on the initiative of the farmer. A hairpin had been swallowed, penetrated through the stomach, and produced an abscess between the diaphragm and the stomach. Two further cows, when examined on 29th October by a veterinary surgeon, acting for the dairy, were found to have abscesses of the udder.

The preceding summary does not lead to any certain conclusion as to the source of the infection, and if it were of human origin this is not surprising. The cases of illness undoubtedly due to this milk varied greatly in character. In some there was only slight sore throat; in others a very severe tonsillitis with acute adenitis; in others a well developed and unmistakable scarlet fever (see chart). Some of the cases of scarlet fever only desquamated partially; while on the other hand some of the cases having only sore throat and no rash had considerable desquamation. In one case the sore throat was followed by rheumatic fever and pneumonia. In another patient (fatal), who at the time she became infected was suffering from gastric ulcer, and therefore living chiefly on milk, the symptoms resembled general septicæmia, pneumonia supervening. In another patient (fatal), so much vomiting followed the sore throat that the patient was suspected to have a perforated gastric ulcer, though this was not so.* If, then, the person infecting the milk in the cowshed was only slightly ill, he might have infected the milk without his illness being recognized. Milk is an excellent medium for the growth of scarlatinal germs. A glance at the chart and at Fig. I. shows that probably the milk of A.'s dairy was infected at intervals to a less extent during the four weeks preceding the great outbreak.

* I have not thought it necessary to enter into further details as to the clinical features of the cases in the epidemic. It may be mentioned that cases of scarlet fever and of sore throat were treated in the scarlatinal wards of our isolation hospital with scarlatinal patients unconnected with this outbreak. No case of cross-infection occurred. Some of the cases lend colour to the supposition that a dual poison was working, and the facts previously stated as to cows' udders may support this view. All the unusual complications present are, however, occasionally found in epidemics of scarlet fever. due to personal infection, and the presence or absence of rash in scarlatinal sore throats is probably in the main a question of dosage of infection. One reason why scarlet fever has not been able to be brought more completely under administrative control is the non-recognition of the scarlatinal nature of sore throats in persons associated with cases of scarlet fever. 
An attempt was made, without success, to trace the milk supplied to the school $\mathrm{Z}$. to a particular cowman, but without success. The foreman at the farm always poured the milk for this school into its special locked can, and no special cowman always milked for this particular supply. The milk milked by the cowman $P$. was mixed with the rest of the milk before leaving the farm.

The main conclusions from these facts are as follows :-

1. The epidemic was due to milk from B.'s farm.

2. Its immediate control was due to the prompt action of the Sanitary Committee, and the active and intelligent co-operation of the dairymen and the farmer.

3. The expense and loss entailed both on the town and on the dairy and farmer by the measures that were taken were far less than would have resulted from the continued supply of infected milk.

I may add that the whole cause of the epidemic illustrated strikingly the advantage to public administration of voluntary co-operation between officials and the sections of the community whose operations have a bearing on public health. This is only one added to several instances given in previous reports, in which satisfactory results have followed on the encouragement given by the Sanitary Committee to such co-operation.

Copy of Circular letter sent to Dairymen in 1905.

The IMPortance OF BEING ABLE TO IDENTIFY THE EXACT SOURCE OF MILK SUPPLY.

Public Health Department,

Town Hall, Brighton,

November 24 th, 1905.

DEAR SIR,

Circumstances have drawn my attention to the difficulty which arises in identifying the original source of milk distributed in the town, especially in the case of large dairies obtaining milk from a number of different farms. It may be within your knowledge that occasionally, though rarely in the experience of Brighton, infections diseases such as scarlet fever, diphtheria, and typhoid fever, have been spread by milk supplied. In order to enable the Sanitary Authority to ascertain the true facts in any case of suspicion, dairymen are under a legal obligation (Section 55 of the Brighton Improvement Act, 1884) to furnish when required a full and complete list of their customers to the medical officer of health. It is obvious, however, that if enquiries were made at each house named on a list thus furnished, some risk would arise of damaging the business of the dairyman, and the above powers have therefore been seldom utilized. When utilized, or even if the necessary information were obtained otherwise, the result would, when infection is traced to a given milk supply, be that the entire supply by a given dairyman or Company would need to be stopped; whereas if the method which I am about to suggest were universally adopted, it would at the worst scarcely ever be necessary to stop the milk from more than one farm, and this could be done with the utmost privacy, without any injury to the trade.

The method to which I allude is one already used at my suggestion by some dairymen in the town, and one which has been found to be of great utility in the interest of the public health, and has obviated serious expense and loss of custom for the dairymen who have employed it. 
The method consists in ear-marking the source of the milk distributed in each round by each milk carrier, and recording the facts day by day. It implies also that the milk from different farms will not be mixed. When this method has been adopted it is easy to place one's finger at once on a source of infection, to visit the farm or other place in which the infection has originated, and by this means to prevent more than a minimum amount of disorganization of business, as well as a minimum amount of infection.

I venture to ask your co-operation in introducing into your business the above method of booking each day the source from which the milk in each milk-round is derived, and thus insuring your business against disturbance, as well as co-operating in preventing the introduction of infection into the town.

\author{
Believe me, \\ Yours faithfully, \\ Medical Officer of Health.
}

\title{
TYPHUS FEVER AND FLEAS.
}

BY PROFESSOR MATTHEW HAY, M.D.,

Medical Officer of Health of Aberdeen.

$T^{\mathrm{HE}}$ following account of an epidemic comprising 131 cases with 1 twenty-two deaths is taken from a report of Professor Hay, reprinted in the Annual Report of the Local Government Board for Scotland, 1906.

The disease was not found to be infectious in its early stages, say, during the first week. In every instance where the first case in a family was removed before the end of the first week of the illness, no other case followed in the family. Different views are expressed by the authorities as to the period of greatest infectiousness, some placing it early and others late, but all appear to be agreed in regarding typhus as one of the most infectious of diseases. For example, Osler, in his well-known work on medicine, after referring to it as one of the most highly contagious of febrile affections, says that "in epidemics nurses and doctors are almost invariably attacked," and further, that "when the sick are aggregated in "wards the poison appears to be concentrated, and the danger of infection is much enhanced." It cannot be doubted that typhus is distinctly infectious ; but the experience of the recent epidemic seems to show, at any rate for that epidemic, that the infectiousness is not so overwhelming as is sometimes believed. Some of the facts just stated tend to bear this out, and there are others. For example, the disease had apparently been in the city for two or three months before it was discovered, and, therefore, before steps were taken to control it, yet it had not produced many cases ; and, later on, when it came under the notice 